\title{
Analysis on the influence of the proportion and growth rate of different categories of drug clinical trial research funds on quality improvement in China recently six years
}

\section{Liran Chen}

Shanghai Ernst\& Young

Huafang Chen ( $\square$ chenhuafang2011@126.com )

Wenzhou Medical University First Affiliated Hospital https://orcid.org/0000-0002-9859-7006

\section{Commentary}

Keywords: inspection of clinical drug trials, cost, quality, SMO, CRC

Posted Date: October 23rd, 2019

DOI: https://doi.org/10.21203/rs.2.12650/v2

License: (c) (1) This work is licensed under a Creative Commons Attribution 4.0 International License. Read Full License 


\section{Abstract}

Background: The China food and drug administration (CFDA) issued the Announcement of Selfexamination and Inspection of Clinical Drug Trial Data on July 22, 2015. Great changes have taken place since the launch of the most stringent drug registration self-examination and inspection in history; the cost of clinical trials is one of the important changes. Methods: The paper compares the changes in the cost of clinical drug trials on both the number and the structure of the trials 3 years before and 3 years after self-examination and inspection were initiated by the CFDA,analyzes the impact on the cost of the clinical research coordinator ( CRC ), the labor service of researchers, audit companies, institutional drug management and quality control on the quality improvement of clinical drug trials. Conclusions: According to the article, the emergence and increase in most clinical trial costs are conducive to the quality enhancement of clinical drug trials,However, the emergence and continued increase of CRC costs can improve the quality of clinical drug trials in some ways and hinder it in others.. To improve the quality of clinical trials, China must regulate the booming site management organization ( SMO ) market and actively formulate industry standards and qualification certifications for CRCs.

\section{Background}

On July 22, 2015, the China food and drug administration (CFDA) issued the Announcement of Selfexamination and Inspection of Clinical Drug Trial Data. Immediately, it opened what the industry called the 7.22 storm. With "the most rigorous standards, the most strict supervision, the most severe punishment, the most serious accountability", the most stringent drug registration self-examination and inspection in history was launched. Great changes have taken place in the field of clinical drug trials with the deepening of self-examination and inspection in China in the past three years, among which the cost of clinical trials is one of the important.

The purpose of this paper is to analyze the change in the quantity and structure of clinical trial costs before and after 7.22 and explore the corresponding relationship between cost change and quality improvement. Both cost and quality changes are the effects of multiple causes, and these two changes initiate further effects. When two kinds of changes interact with each other, there is a positive correlation or interdependence between the changes. However, in reality, the increase of expenses and the improvement of quality do not correspond to each other. It is possible that with the increase of investment, the competition for benefits in some aspects may go against the improvement of quality.

\section{Methods}

\section{Changes in the cost of clinical trials before and after 7.22}

There are different visit procedures and difficulty levels in different clinical drug trial protocols. In recent years, diabetes and cancer drugs have been frequently involved in clinical trials because the input costs of similar drugs are comparable. For example, we can analyze the change in the amount and 
composition of the clinical trials cost. In this paper, the data come from the First Affiliated Hospital of Wenzhou Medical University, Tianjin Medical University General Hospital and Wuhan Union Hospital. Since multi-center clinical drug trials are generally signed contracts at the same price at every center, the three hospitals can represent the average cost level over the period in China.

This paper focuses on the costs involved in the agreements signed by the hospitals during innovative drug phases II and III and generic drug random controlled clinical trials and analyzes the monitoring, audit and tax input of the sponsor. It does not involve the costs of phase I clinical trials, clinical trials data management statistics, cold chain logistics or researcher meetings. The unit of measurement is RMB yuan.

\subsection{Change trend of clinical trial costs from 2013 to 2018 (taking the drug for glycemic control as an example)}

The paper selects programs with similar visiting frequencies and observation periods and compares the costs of the clinical trial agreements signed three years before and three years after July 22, 2015. (Note: in the chart below, the cost for 2015 is before July 22; for approximately half a year after 7.22, the overwhelming majority of Chinese hospitals endured a shock period in terms of contract signing).

\subsubsection{The change in the global clinical trial cost during 2013-2018}

Fig. 1 shows the cost and composition of clinical trials for innovative drugs for glycemic control from the United States and European countries in agreements signed by hospitals in the territory of China from 2013-2018. The most significant change in absolute value and proportion is paid to the clinical research coordinator ( CRC) of the SMO (site management organization). From 2013-2018, the average amounts paid were $0,0,0,8052,13,154$, and 14,865 , respectively, presenting remarkable changes before and after 7.22 and indicating an increasing trend year by year. The average investigator fee from 2013 to 2018 was $21,312,22,086,21,830,24,055,23,898$ and 26,219 , respectively, for which the general change is not obvious, and the slight increase is related to inflation. The average number of research subjects submitted to laboratory and imaging examination in 2013-2018 was 935, 968, 985, 992, 987 and 997, respectively, which barely changed over the period because the examination fee was set, the hospital charge standard was approved by the price department, and the charge standard change did not happen in these years. The average amount of transportation subsidy from 2013 to 2018 was 1150, 1097, 1116, 1235,1184 and 1183 , and the transportation subsidy was usually between 150 and 200 for a single visit. The document management fee remained unchanged for all 6 years at 2000 per case for 15 years. Since July 22,2015 , the drug management fee has been 500 per case, which entails an obvious but small change in amount.

\subsubsection{The change in Chinese clinical trial cost during 2013-2018}

Fig.2 shows the cost and composition of clinical trials for generic drugs for glycemic control in agreements signed by hospitals in the territory of China during 2013-2018. The most significant change 
of absolute value and proportion is paid to the CRC of the SMO. From 2013-2018, the average amounts of $0,0,0,3530,5065$ and 8120 , respectively, presented remarkable changes before and after 7.22 , with an increasing trend year by year. The average investigator fee from 2013 to 2018 was 5800, 5675, 6035, $9126,11,112$ and 11,293 , respectively, with a clear general change. The average number of research subjects submitted to laboratory and imaging examination in 2013-2018 was 965, 983, 968, 972, 969 and 991 , which barely changed because the examination fee is set, and changes proposed by hospitals are approved by the price authority. The average subject transportation subsidy from 2013 to 2018 is 0,200 , $330,650,950$ and 960; the cost of a single visit was not counted, and either a little fee was paid when the clinical trial was completed or there was no transportation subsidy before 7.22. The transportation subsidy for each visit ranged from 100 to 150 after 7.22. The fee for document management was not counted. The drug management fee was 500 per case after July 22, 2015.

Comparing Fig.1 and Fig.2, the investigator fee and cost to the CRC in a multi-center clinical trial are significantly higher in global clinical trials than in China clinical trials. In addition to the difference between sponsors' economic power, multi-center clinical trials around the world focus on innovative drugs, while the majority of Chinese clinical trials concern generic drugs.

\subsection{Composition and proportion of clinical trial costs 3 years before and after 7.22 (taking lung cancer drugs as an example)}

Cancer drugs are common subjects of clinical drug trials in recent years, so the cost of cancer drug clinical trials is representative. We take the cost of lung cancer drugs, which includes 18 to 20 visits excluding the lifetime visits and unscheduled visits; for example, compare the cost of clinical trials before and after July 22, 2015.

\subsubsection{Composition and proportion of the costs of clinical trials with global trials sponsors}

\subsubsection{Composition and proportion of costs of clinical trials with global trials sponsors within China 3 years before July 2015}

The average fees from 2013-2015 are shown in Fig.3, as follows: the investigator fee was 30,697, or $64.09 \%$ of the proportion; the subject examination fee was 11,847 , or $24.73 \%$ of the proportion; the subject transportation subsidy was 3355 , accounting for $7.00 \%$; the document management fee was 2000 , accounting for $4.18 \%$.

\subsubsection{Composition and proportion of costs of clinical trials with global trials sponsors within China 3 years after July 2015}

The average fees from 2016-2018 are listed in Fig.4, as follows: the investigator fee was 40,187, or $44.37 \%$ of the proportion; the subject examination fee was 12,053 , or $13.31 \%$ of the proportion; the subject transportation subsidy was 4200 , accounting for $4.64 \%$; the document management fee was 2000 , accounting for $2.21 \%$; the drug management fee was 500 , accounting for $0.55 \%$; and the CRC fee was 31,624 , accounting for $34.92 \%$. 


\subsubsection{Composition and proportion of Chinese clinical trial costs}

\subsubsection{Composition and proportion of the costs of Chinese clinical trials within China 3 years before July 2015}

Fig.5 shows the average fees from $2013-2015$, as follows: the investigator fee was 18,930 , or $71.91 \%$ of the proportion; the subject examination fee was 5395 , or $20.49 \%$ of the proportion; the subject transportation subsidy was 2000 , accounting for $7.60 \%$; and no document management fee was charged.

\subsubsection{Composition and proportion of the costs of Chinese clinical trials within China 3 years after July 2015}

The average fees from 2016-2018 are shown in Fig.6, as follows: the investigator fee was 23,400, or $47.85 \%$ of the proportion; the subject examination fee was 6252 , or $12.78 \%$ of the proportion; the subject transportation subsidy was 2250 , accounting for $4.60 \%$ the drug management fee was 500 , accounting for $1.00 \%$; the CRC fee was 16,500 , accounting for $33.74 \%$; and no document management fee was charged.

Comparing Fig. 1 with Fig. 3 and Fig. 4 for clinical trials with global trials sponsors or comparing Fig. 2 with Fig. 5 or Fig. 6 for Chinese clinical trials, the composition and proportions of the cost are similar for the clinical trials for glycemic control drugs and cancer drugs. One difference is that the clinical trials for cancer drugs involve more imaging and laboratory examination, which have higher costs and represent a larger proportion of the fees.

\subsection{Analysis of the cost change}

\subsubsection{Investigator fee}

The increase in the investigator fee of global clinical trial cost is not obvious, and eliminating inflation, the amount shows little change in Fig.1. Fig.2 shows that the cost of the Chinese multi-center site grew notably by $30-40 \%$ after 7.22 .

\subsubsection{CRC fee}

The CRC fee shows the most significant change. There were few CRC fee expenditures before July 22, 2015, but fees increased year by year from 2016 to 2018. As shown in Fig.1, the amount and proportion of CRC fees associated with multi-center clinical trials for glycemic control drugs from 2016-2018 were 8052 (22\%), 13154 (32\%) and 14865 (32\%), respectively. As shown in Fig. 2, the amount and proportion of CRC fees associated with the Chinese multi-center clinical trials for glycemic control drugs from 20162018 were 3530 (24\%), 5065 (27\%) and 8120 ((37\%). Fig.4 shows that the mean CRC fee for multi-center clinical trials for cancer drugs global from 2016 to 2018 was 31,624 , and the proportion was $34.92 \%$. 
Fig. 6 shows that the mean CRC fee associated with the Chinese multi-center clinical trials for cancer drugs from 2016 to 2018 was 16,500, and the proportion was $33.74 \%$.

\subsubsection{Drug management fee}

7.22 promoted the standardized management of drugs in good clinical practice (GCP)central pharmacies and the process of clinical drug trials; due to the low amount of this fee, it represented only approximately $1 \%$ of the total investment in clinical drug trials,this article does not foucus on drug management fee.

\subsubsection{Document management fee}

There are some differences between hospitals in terms of Chinese and global clinical trials. According to the Good Clinical Practice of Pharmaceutical Products [SFDA order No. 3, Effective on September 1, 2003], Article 52, "The investigator shall keep the documents of the clinical trial for five years after the completion of the trial." ${ }^{1}$ However, after the completion of clinical drug trials with global trials sponsors, the documents generally must be kept for 15 years. While the documents from global trials are preserved freely for all 15 years, in China, after the first 5 years following a trial's completion, hospitals charge 15005000 every year for the 6th to the 15th years, on the basis of the case amount and number of documents. After 5 years, the documents from completed clinical trials in China can be handed over to a qualified third party specified by the sponsor; in such cases, although hospitals continue to store the documents for inspection, they rarely charge a fee.

\subsection{Related cost changes not covered by the clinical trial agreements before and after 7.22}

Change is obvious in terms of monitoring and auditing, which mainly concern the input of human activity. After 7.22, along with rigorously CFDA inspections of concomitant drugs, adverse events (AE) and serious adverse events ( SAE) need to be monitored in hospital information system(HIS), laboratory information systems (LIS) and picture archiving and communication systems (PACS) to ensure that a combination drug, especially a combination drug prohibited by the protocol, AEs and SAEs are recorded completely and without omission. In recent years, the risk-based monitoring advocated by the sponsor has improved the monitoring efficiency to some degree, but in general, after $7.22,15-20 \%$ more time is spent monitoring in the hospital, and the corresponding labor cost increases by $15-20 \%$.

After 7.22, the attention sponsors pay to auditing has significantly increased, and the frequency of audits of hospitals has increased significantly. Generally, the sponsor pays the fees and entrusts a third-party audit company to carry out the audit. Another change concerns the tax. Because the State Administration of Taxation changed the value added tax rules two years ago in China, and the clinical trial cost in the invoice billing system was within the scope of the tax, when sponsors remit money to hospitals, the added $6 \%$ tax must be paid. Thus, there was a $6 \%$ increase in the total amount of money the sponsor had to pay. The tax cost has nothing to do with the 7.22 event, but the universal implementation of the tax law reform occurred close to 7.22 . 


\section{Results}

\section{Industry changes and cost changes after 7.22}

After 7.22, both the global and Chinese domestic multi-center clinical trials in China changed, and the change in clinical trials initiated by Chinese domestic sponsors is greater than the change in clinical trials with global trials sponsors.

2.1 A new phenomenon in the industry in the past three years: the emergence of SMOs and audit companies and the increase in clinical trial prices

\subsubsection{Increase in SMOs}

An SMO provides study coordination services and sends a CRC to the hospital to assist in the implementation of the clinical trial and perform the nonmedical judgment duties authorized by the principal investigator. SMOs began to appear in 2008 in China and remained small scale until July 22 , 2015. Then, the number of SMOs increased rapidly and the number of employees of SMOs also increased. For example, an SMO grew to nearly 600 CRCs within one year after its establishment.

\subsubsection{Centralized management of drug trials at the center pharmacy}

It is difficult for departments of internal and surgical departments undertaking clinical trials to manage trial drugs by specially-assigned personnel, and their medical staff do not have sufficient experience in trial drug management. In addition the limited equipment used to store drugs for clinical trials in Internal medicine, surgery, pediatrics and other clinical departments do not meet the inspection requirements for undertaking clinical trials. The GCP center pharmacy provides centralized management by full-time pharmacists who pass GCP training and can better perform the standard operating procedures of trial drug reception, storage, distribution, use, residual drug recycling and destruction, etc.

\subsubsection{Emergence of audit companies}

After 7.22, third-party audit institutions sprang up successively, providing clinical trial training, consulting services, and comprehensive on-site auditing of clinical trial data and records. The independent examination of these audit companies refines and specializes the division of labor.

\subsection{Increase in costs promoted by industry change}

\subsubsection{Increase in CRC fees}

The increase in CRC fees was the most rapid and significant change associated with the cost of clinical trials, which is clearly shown in Figures 1-6.

\subsubsection{Investigator fee}


The increase in the investigator fee is divided into explicit growth, which can be clearly shown in Figures 1-6, and implicit growth. Implicit growth refers to the addition of the CRC, in that part of the nonmedical judgment work that was previously done by the principal investigator is currently done by the CRC. The investigators' labor fees have increased in disguised form because of the decrease in their workload.

\subsubsection{Audit company fees}

The audit company usually sends 5 persons to a site for 2 to 3 days for an on-site audit, and the sponsor needs to pay the audit company a fee of 80 to 100 thousand yuan. The number of sites that are audited is determined by the evaluation of sponsor and the request of the clinical trial site.

\subsubsection{Drug management fee and quality control fee}

The drug management fee is small, and each subject is generally charged a fee of 200-500 yuan according to the complexity of the clinical trial; this method has played a positive role in regulating the management of drugs in the implementation of clinical drug trials. Before 7.22, the clinical drug trial research institutions charged lower quality control fees, and some institutions began charging quality control fees after 7.22. The fees charged by agencies vary, and the overall amount is small. The fees are earmarked for this specified purpose and therefore play a role in quality control.

\section{Discussion}

There is a saying in the industry about the cost and quality of clinical trials: high costs do not necessarily mean high quality, but low costs certainly cannot yield high quality. The consensus in the industry is that low cost cannot guarantee the quality of clinical trials, and any step requires a budget for labor and material resources. Research institutions connot perform high-quality clinical trials for a sponsor with a limited budget. On the other hand, there are many factors influencing quality. High investment and high cost do not necessarily guarantee high quality.

The price of clinical trials showed a sharp increase over nearly 3 years. The quality of clinical trials also undergoes obvious changes, but there is no direct correspondence between them. Both of these changes may share one cause and cause multiple subsequent effects, or a change may have multiple causes and one effect. A price increase is necessary for quality improvement, but it is not a sufficient condition. Price increase is not positively correlated with quality improvement, and certain factors that raise prices can improve quality in some ways while obstructing quality improvement in other ways.

\section{Conclusions}

\section{The growing SMO companies need to be regulated}

Most of the cost increases in clinical trials are positively correlated with quality improvements, but the increase in costs caused by the booming development of SMO and the improvement in the quality of 
clinical trials should be viewed in two aspects The CRC service fee paid by the sponsor to the SMO accounts for approximately $30 \%$ of the total investment in clinical trials, which cannot be underestimated. The intervention of the $\mathrm{CRC}$ has undeniably provided convenience and some assistance for investigators to improve the quality of clinical trials. However, there are also disadvantages associated with the deficient abilities of the $\mathrm{CRC}$, the investigator absence of the for the management of clinical trials and the excessive dependence of the investigator on the CRC.

\subsection{Absence and disadvantages associated with the CRC}

After 7.22, the research institutions that undertake clinical drug trials asked CRCs to facilitate trials at the time of signing the agreement with the sponsor or contract research organization (CRO). The CRC agreement is a separate triple agreement is generally independent from the main clinical trial agreement and is signed by the sponsor (or CRO), SMO and the hospital (research institution). Since the financial management of Chinese public hospitals does not allow payment to be easily sent to businesses such as SMOs, it is stipulated in the triple agreement that the sponsor or CRO shall remit money to the SMO, and the SMO shall send a CRC with a relevant professional background and GCP training to the hospital to assist with the investigator's work. The CRC must sign a confidentiality agreement concerning the work content when the principal investigator authorizes and assigns the CRC tasks of nonmedical judgment. The persons responsible for the work content performed by the CRC are principal investigators, subinvestigators $\llbracket$ when the CRC is incompetent, hospitals (research institutions) have the right to require the SMOs to replace him or her with a competent CRC.

The lack of industry training and supervision impedes the rapid development of the SMO industry. Newly registered SMOs have sprung up, and the shortage of CRC talent has led to a war between SMO companies. CRCs frequently job hop, and an impetuous atmosphere is spreading within the industry, which results in a profit-oriented short-term development model among SMOs. The training process before a new CRC takes up the post can be shortened or even omitted. Another problem is that the management of CRCs by SMOs is regulated via long-distance supervision. The CRCs work in various hospitals in different districts, and the SMO functions in the registration district, which also makes supervision difficult.

The majority of CRC practitioners are mostly undergraduate or even community college graduates in pharmacy or nursing, and their professional skills are poor. In addition, most of them enter the CRC industry immediately after graduation, with no working experience and insufficient professional knowledge accumulation. In addition to the inadequate training of SMO companies $₫$ many incompetent CRCs are sent to hospitals to perform CRC functions.

From the perspective of the hospital, the investigators consider themselves very lucky if an excellent CRC is sent to their site; a CRC of average quality is normal; and even a incompetent CRC is better than none. In other words, the investigators dare not expect a good CRC, considering that any CRC is better than none. 
While the CRCs have low-quality skills, they have plenty of time to assist the investigators; on the other hand, the investigators are too busy with clinical work and his patients to focus on the clinical trials. In such a state, CRCs perform the duties of many of the investigators and even the principal investigators. The principal investigators' work is to sign for authorizations, then engage in clinical work, hand over the clinical trial work to $\mathrm{CRC}$, When the clinical trial forms is need to be signed during the subsequent trial,these forms are all signed by the primary investigator and the investigator after the CRC has filled in and prepared. Most of the time, the contents of the signed forms are not asked before the primary investigator and the investigator hurriedly sign them.Another common phenomenon concerns the CRCs' use of the investigators' account and password authorized by the sponsor for logging into the electronic date capture system( EDC). when CRC used the investigators ' account and passwords to fill out numerous forms, the investigators ignored the review before the investigator and primary investigator signed it,so the process and date completed by the CRCs have not beenveritied by investigators Blind signatures, error-ridden process and content and the investigators' leaking of EDC account passwords to CRCs mean that the monitoring of the clinical trials may not cover the whole process and entail hidden dangers in clinical drug trials.

\subsection{Sponsors and clinical trial institutions kidnapped by SMOs}

A hospital or clinical trial institution requires a sponsor to provide a CRC for a clinical trial; the sponsor remits the funds and entrusts the task to an SMO; and the SMO provides CRC services and sends a CRC to the clinical trial site. The long-distance supervision of the CRC by the SMO works poorly, and there is a lag in the evaluation of CRC work quality in clinical trials site..In that situation, timely and effective feedback is lacking. Once a CRC's incompetence is discovered, the effects on the quality of the clinical trial and the trial schedule are irreversible. The long-distance supervision by the sponsor and the SMO cannot effectively control the quality of CRCs. If the hospital office managers and investigators of clinical trials find that a CRC does not meet the quality requirements and a replacement of CRC is required, the hospital staff must consider the time and opportunity costs of CRC turnover. There are risks. For example, the replacement CRC may also be incompetent; it takes time for the new CRC become familiar with the work; interactions with different CRCs may also lead to mutual buck-passing due to the work handover when problems arise. The current shortage of CRCs and scarcity of high-quality CRCs have led SMOs to "kidnap" bidders in terms of service price and "kidnap" hospitals and investigators in terms of service quality.

\subsection{Exploring new CRC models}

In addition to the SMOs providing CRCs, individual hospitals, such as Zhongshan Hospital Affiliated of Fudan University, Beijing 301 Hospital, Hunan Cancer Hospital and Cancer Hospital Affiliated of Sun Yatsen University, established their own CRC teams to assist with clinical trials. Their purpose is to create a high-level and stable CRC team that can assist investigators in accomplishing high-quality clinical drug

trials, turning from the model of outsourcing CRC services from SMOs to the model of training, employing and supervising CRCs within the hospital itself. The hospitals' own CRCs account for a small proportion 
of the total number of CRCs currently employed by SMOs. Each model for supplying CRCs, either outsourcing from SMOs or providing CRCs within the hospital, has advantages and disadvantages. CRCs sent by SMOs can cover clinical trial sites in cities across the country, but the personnel sent by SMOs to hospitals need to undergo a process of familiarization and be acceptanced by hospitals. A CRC team within a hospital has many advantages in undertaking local work, but it cannot support the clinical trial needs of other hospitals.

There are no universally recognized regulations on CRCs in current China, and some professional associations are actively formulating industry standards and qualification certifications for CRCs. For example, the Professional Committee of Pharmaceutical Clinical Trials of Guangdong put forward the Guangdong consensus on CRC management, and the Alliance of Pharmaceutical Clinical Trial Institutions issued CRC industry guidelines. Despite the efforts of the sponsors, hospitals and even SMOs, there are no clear and commonly accepted operational standards.

From a positive perspective, 7.22 has opened up a new situation of quality requirements for clinical trials, but much remains to be accomplished in the gradual and ongoing process of improving clinical drug trials. Only cooperation and continuous collaborative efforts can lead to progress.

\section{Abbreviations}

SMO Site Management Organization

CRC Clinical Research Coordinator

GCP Good Clinical Practice

CFDA China Food and Drug Administration

AE Adverse Events

SAE Serious Adverse Events

HIS Hospital Information System

LIS Laboratory Information System

PACS Picture Archiving and Communication Systems

CRO Contract Research Organization

EDC Electronic Date the Capture System

\section{Declarations}




\section{Ethics approval and consent to participate}

The name of the ethics committee: The Clinical Research Ethics committee of the First Affiliated Hospital of Wenzhou Medical University

Committee reference number: Not applicable

Our manuscript does not report on or involve any animals, humans, human data, human tissue or plants.

\section{Consent for publication}

Not applicable

\section{Availability of data and material}

The datasets analyzed during the current study are available from the corresponding author on reasonable request.

All data generated or analyzed during this study are included in this published article .

\section{Competing interests}

The authors declare that they have no competing interests.

\section{Funding}

No funding was obtained for this study.

\section{Authors' contributions}

Huafang Chen analyzed and interpreted the study data. Liran Chen was a major contributor in writing the manuscript. Both authors read and approved the final manuscript.

\section{Acknowledgements}

Not applicable

\section{References}

1 (2003). Good Clinical Practice of Pharmaceutical Products, China State Food and Drug Administration

\section{Figures}




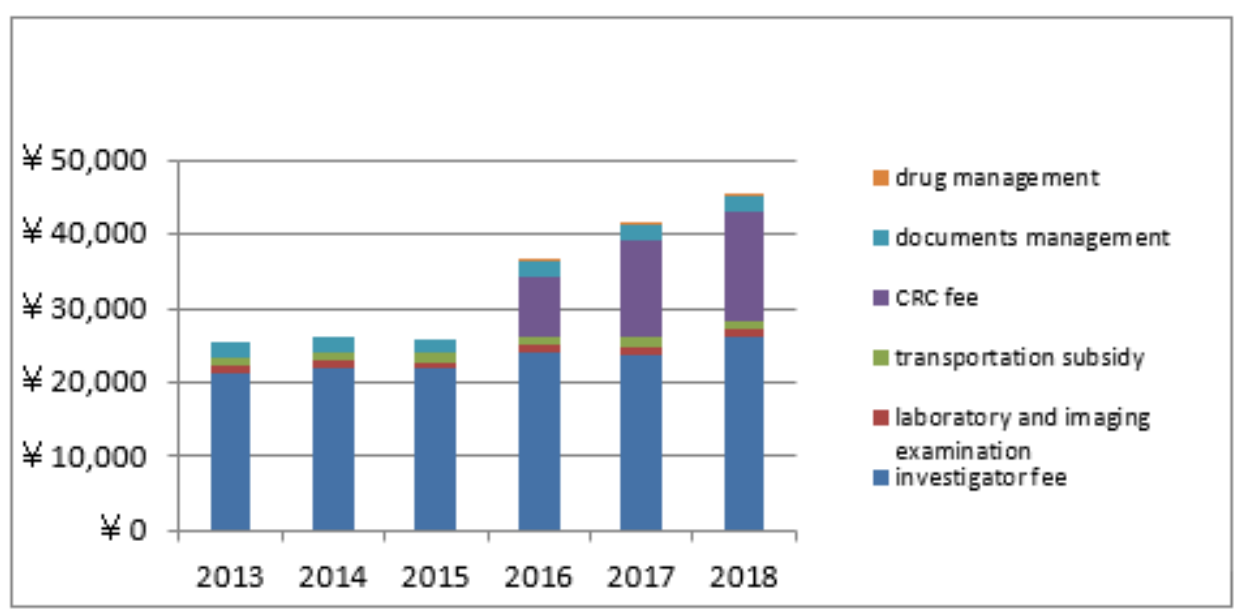

\section{Figure 1}

2013-2018 year innovative glycemia controlling drugs cost amounts and composition of global clinical trial

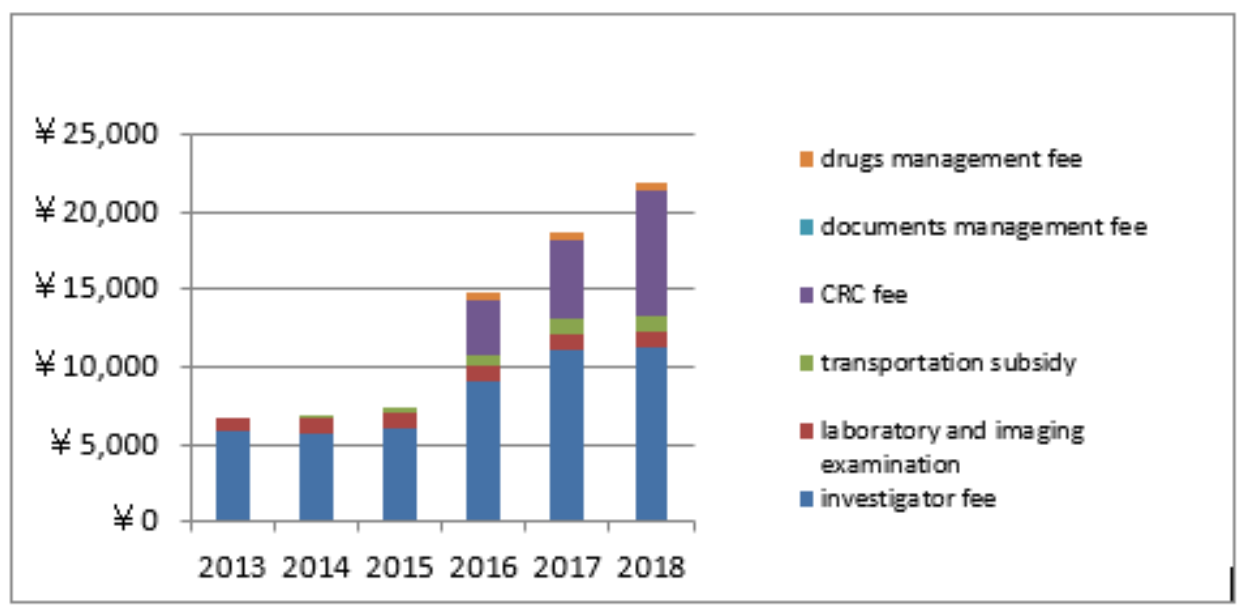

\section{Figure 2}

2013-2018year generic glycemia controlling drugs cost amounts and composition of China clinicl trial

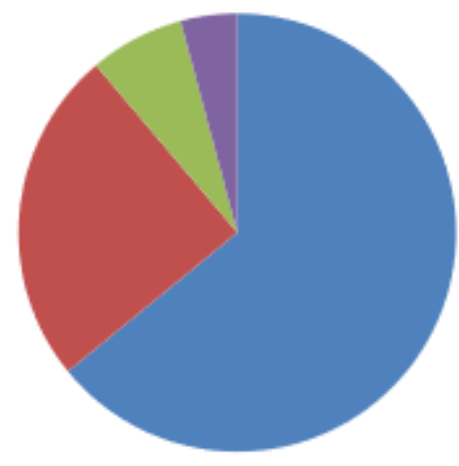

Investigator fee $64.09 \%$

Investigator fee $24.73 \%$

subjects traffic subsidy $7.00 \%$

documents management $4.18 \%$ 
Figure 3

2013-2015 composition and proportion of global clinical trial lung cancer cost

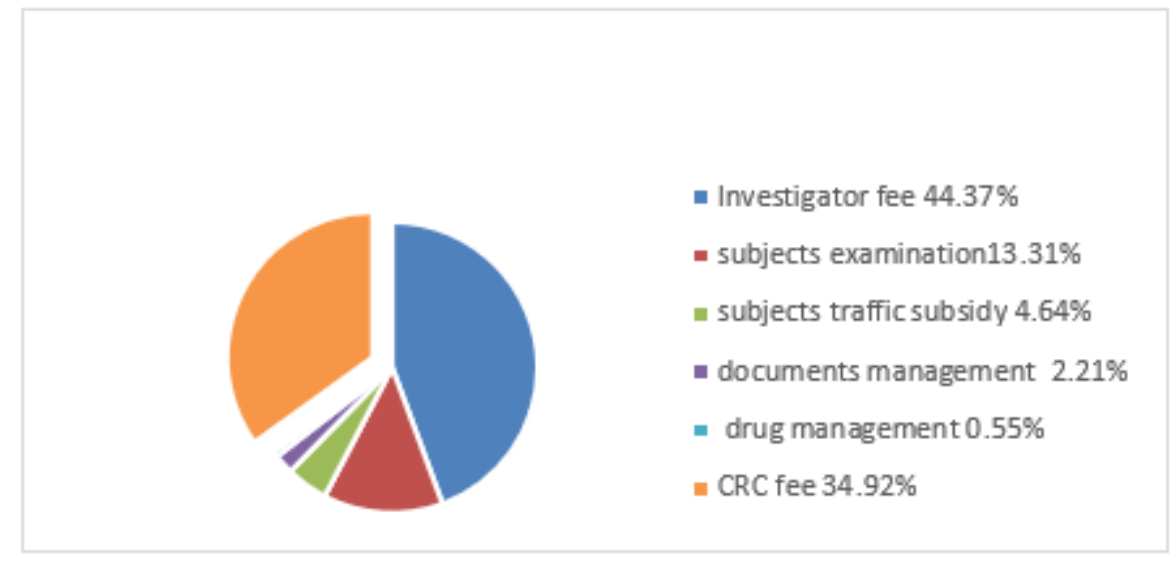

\section{Figure 4}

2016-2018 composition and proportion of global clinical trial lung cancer cost

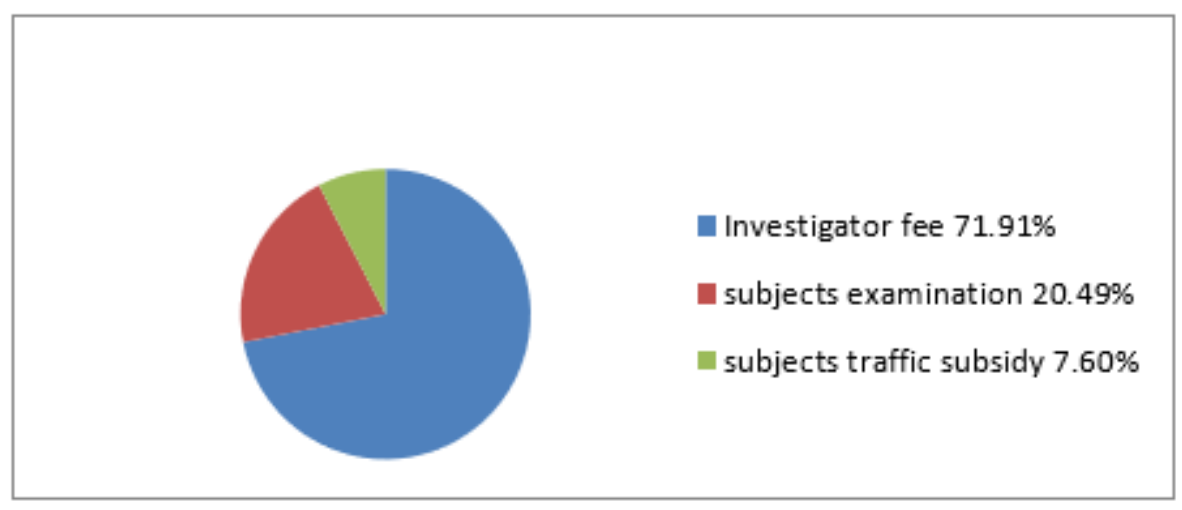

\section{Figure 5}

2013-2015 composition and proportion of Chinese clinical trial lung cancer cost

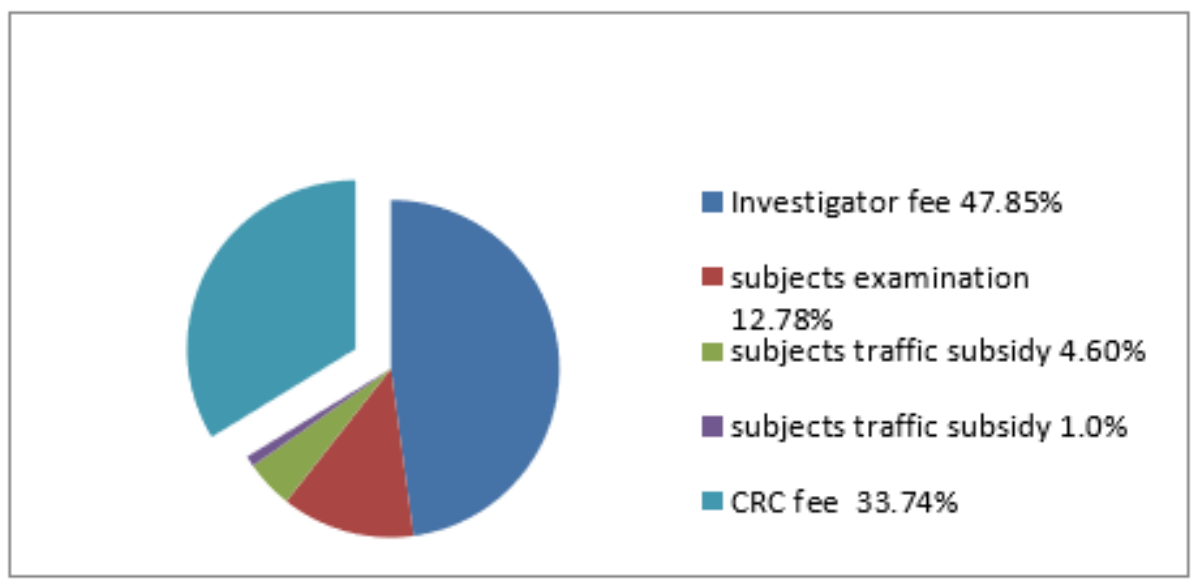

\section{Figure 6}


2016-2018 composition of Chinese clinical trial lung cancer cost

\section{Supplementary Files}

This is a list of supplementary files associated with this preprint. Click to download.

- EQUATORNetworkReportingChecklist.docx 